\title{
Bose-Einstein condensation with magnetic dipole-dipole forces
}

\author{
Krzysztof Góral†, Kazimierz Rzążewski† and Tilman Pfau $\S^{b}$ \\ $\dagger$ Center for Theoretical Physics and College of Science, Polish Academy of Sciences, \\ Aleja Lotników 32/46, 02-668 Warsaw, Poland \\ $\S$ Faculty of Physics, University of Konstanz, 78457 Konstanz, Germany
}

(September 2, 2021)

\begin{abstract}
Ground-state solutions in a dilute gas interacting via contact and magnetic dipole-dipole forces are investigated. To the best of our knowledge, it is the first example of studies of the Bose-Einstein condensation in a system with realistic long-range interactions. We find that for the magnetic moment of e.g. chromium $\left(6 \mu_{B}\right)$ and a typical value of the scattering length all solutions are stable and only differ in size from condensates without long-range interactions. By lowering the value of the scattering length we find a region of unstable solutions. In the neighborhood of this region the ground state wavefunctions show internal structures not seen before in condensates. Finally, we find an analytic estimate for the characteristic length appearing in these solutions.
\end{abstract}

Since the advent of Bose-Einstein condensation in dilute gases of alkalies [1] and hydrogen [2] it has become apparent that the interactions between the condensed atoms govern most of the observed phenomena. In all the experiments so far the interaction can be described by a contact potential which is characterized by the scalar quantity $a$ being the s-wave scattering length. Static properties like the condensate's ground-state density profile, its instability in the case of negative $a$ and the meanfield shift in spectroscopic measurements as well as dynamic properties like collective excitations and propagation of sound have been investigated [3]. Similarly, nonlinear atom optics experiments e.g. four wave mixing [4] are only possible due to the large nonlinearity mediated by the atom-atom interactions. The $T=0 \mathrm{~K}$ situation in almost all experiments can be described very well by the Gross-Pitaevskii equation [5].

Any reasonably strong dipole-dipole interaction would largely enrich the variety of phenomena to be observed in dilute gases due to their long range and vectorial character. However, for all of the condensed atomic species the magnetic moment $\mu$ was roughly $1 \mu_{B}$ (Bohr magneton) and the respective magnetic dipole-dipole interaction was negligible compared to the contact potential. It has been proposed to induce a strong electric dipoledipole interaction in alkalies by the application of strong DC electric fields [6].

Recently it has become possible to trap atoms with higher magnetic moments at high densities. Examples are europium $\left(\mu=7 \mu_{B}\right)$ [7], which has been trapped magnetically by a buffer-gas loading technique, and chromium $\left(\mu=6 \mu_{B}\right)$, which has been loaded into a mag- netic trap by a buffer-gas technique [\$ and by laser cooling [9]. For these species the scattering lengths are not known to date, but, assuming a normal non-resonant behavior, the crossections of the scalar contact potential and the magnetic dipole-dipole interaction are of comparable size.

As the dipole-dipole interaction is attractive parallel to a common polarization axis the immediate question arises: can a stable condensate be formed under the influence of a dipole-dipole interaction? What is its effect on anisotropic clouds? What do the ground-state wavefunctions look like? In this Letter we address these questions and choose the magnetic dipole-dipole interaction in a dilute cloud of chromium atoms as an example for numerical calculations. The results however apply for all static dipole-dipole interactions.

In general, the Gross-Pitaevskii equation with a longrange interaction term and for a cylindrical harmonic trap has the following form:

$$
\begin{gathered}
i \hbar \frac{\partial \Psi}{\partial t}=\left\{-\frac{\hbar^{2} \triangle}{2 m}+\frac{1}{2} m \omega_{0}^{2}\left(x^{2}+y^{2}+\gamma^{2} z^{2}\right)+\right. \\
\left.+\frac{4 \pi \hbar^{2} a}{m} N|\Psi|^{2}+N \int V\left(\vec{r}-\overrightarrow{r^{\prime}}\right)\left|\Psi\left(\overrightarrow{r^{\prime}}\right)\right|^{2} d^{3} \overrightarrow{r^{\prime}}\right\} \Psi
\end{gathered}
$$

where $\Psi$ is the mean-field condensate wavefunction, $a-$ the s-wave scattering length, $N$ - number of atoms and $m$ - mass of the atom. The reference frequency of the trap $\omega_{0}$ is chosen in the $x y$ plane and the anisotropy of the trap is defined by the $\gamma$ factor. In our case, the longrange potential takes the form characteristic of magnetic dipole-dipole interactions:

$V\left(\vec{r}-\overrightarrow{r^{\prime}}\right)=\frac{\mu_{0}}{4 \pi} \frac{\overrightarrow{\mu_{1}}(\vec{r}) \cdot \overrightarrow{\mu_{2}}\left(\overrightarrow{r^{\prime}}\right)-3 \overrightarrow{\mu_{1}}(\vec{r}) \cdot \vec{u} \overrightarrow{\mu_{2}}\left(\overrightarrow{r^{\prime}}\right) \cdot \vec{u}}{\left|\vec{r}-\overrightarrow{r^{\prime}}\right|^{3}}$,

where $\vec{u}=\frac{\vec{r}-\overrightarrow{r^{\prime}}}{\left|\vec{r}-r^{\prime}\right|}$ and $\mu_{0}$ is magnetic permeability of the vacuum. We will assume that all the magnetic moments are in the same direction $\left(\overrightarrow{\mu_{1}}=\overrightarrow{\mu_{2}}\right)$, which will be referred to as the polarization direction. Note that, depending on a configuration of dipoles, magnetic potential can be repulsive as well as attractive. This fact is a source of a variety of phenomena which do not appear in condensates with contact interactions only. Another peculiar feature of this long-range (integral) term is that for a uniform density distribution it vanishes if integrated within a sphere, which means that in that case a dipole placed in the middle of the sphere would feel no magnetic force. 
Equation (11) is now an integro-differential equation. The integral term can be simplified, because a part of it can be calculated analytically. First of all, one should notice that this term has a form of a convolution:

$$
\int V\left(\vec{r}-\overrightarrow{r^{\prime}}\right)\left|\Psi\left(\overrightarrow{r^{\prime}}\right)\right|^{2} d^{3} \overrightarrow{r^{\prime}}=V(\vec{r}) *|\Psi(\vec{r})|^{2}
$$

The transform of the potential reads:

$$
\mathcal{F}(V(\vec{r}))=\mu_{0} \mu^{2}\left(1-3 \cos ^{2} \alpha\right)\left[\frac{\cos (b q)}{(b q)^{2}}-\frac{\sin (b q)}{(b q)^{3}}\right]
$$

where $b$ is a distance below which atoms overlap (i.e. the radius of the atoms which is of the order of a few Bohr radii) and $\alpha$ is the angle between the Fourier variable $\vec{q}$ and $\vec{\mu}$. A value of $b$ is small in comparison with a length scale in the system (oscillator unit) and we will not consider large values of $q$ therefore it is sufficient to use the limit:

$$
\lim _{b \rightarrow 0} \mathcal{F}(V(\vec{r}))=-\frac{1}{3} \mu_{0} \mu^{2}\left(1-3 \cos ^{2} \alpha\right) .
$$

In order to evaluate the Fourier transform of eq. (3) $\mathcal{F}\left(|\psi|^{2}\right)$ is calculated numerically (FFT) and multiplied by $\mathcal{F}(V(\vec{r}))$ which depends solely on $\alpha$.

In order to obtain the ground-state solution for the condensate one has to solve the Gross-Pitaevskii equation in the form of Eq.(1). To do it we employ a variant of the split-operator method called the imaginary-time propagation method, being now a common routine of solving the Gross-Pitaevskii equation. For each time-step one needs to compute four FFT's: two for the long-range term calculation and two for the evolution.

Now we will list parameters used in the calculations. The reference trap frequency $\omega_{0}$ is equal to $2 \pi 150 \mathrm{~Hz}$ which is an accessible and typical value in present experiments. This corresponds to a characteristic length unit in the system (oscillator unit) $d=\sqrt{\frac{\hbar}{m_{C r} \omega_{0}}} \simeq 1.14 \mu \mathrm{m}$. The s-wave scattering length for chromium is unknown - instead a value for sodium is assumed tentatively $a=a_{N a}=2.75 \mathrm{~nm}$.

One should keep in mind that all the presented solutions are scalable. For the Gross-Pitaevskii equation with as well as without long-range forces the solutions stay the same - in scaled coordinates - as long as the product $N \sqrt{\omega_{0}}$ is kept constant.

The ratio of the contact and long-range terms in general is given by $\frac{\mu^{2} m}{a}, m$ being the atom's mass, and solutions stay identical if it is kept constant. For the chosen example of chromium we will in the second part of the Letter vary the scattering length to change that ratio.

The main conclusion is that, for this set of parameters (in particular the scattering length value) all the investigated solutions are stable, even in very asymmetric traps. Moreover, the wavefunctions possess familiar shapes: Gaussian-like for a small number of atoms and parabolic-like for bigger condensates.
At first, we investigated two extreme cases in cylindrical traps. Following simple intuition about an interaction of two dipoles, we designed these situations so that in the first one there would be a majority of repulsive forces, whereas in the other one an attractive component would be dominant.

In the first situation, the trap is flattened in a plane perpendicular to the polarization axis ( $x y$ plane) with the asymmetry factor $\gamma=\frac{\omega_{z}}{\omega_{0}}=10$ (referred to as discshaped). For this, and only this case, we lower the reference frequency to $\omega_{0}=2 \pi 15 \mathrm{~Hz}$ to make $\omega_{z}=10 \omega_{0}$ experimentally accessible (the corresponding oscillator unit is $d=\simeq 3.6 \mu \mathrm{m})$. In the inset of Fig.1 half-width of the ground state vs. number of atoms is plotted for the disc-shaped trap. It is compared to a corresponding half-width for a Thomas-Fermi solution for a condensate with contact interactions only (referred to as the standard Thomas-Fermi limit). Note that the Thomas-Fermi approximation is good only for large number of atoms, thus for $N<10,000$ it has not been used. In the $z$ direction the exact solution is indistinguishable from the standard Thomas-Fermi limit, because $z$ is a stiff direction and so all the interparticle interaction effects are small with respect to the trapping forces. On the other hand, along the $x$-axis the condensate expands with respect to the standard Thomas-Fermi limit as it should be the case for the repulsive direction. To conclude, in the disc-shaped trap the net effect of interactions is repulsive and the condensate expands.

The opposite extreme case is a trap stretched along the polarization axis ( $z$-axis) with $\gamma=0.1$ (referred to as cigar-shaped) - see Fig.1. For the soft direction (along the $z$-axis, being also an attractive direction), in agreement with simple intuition, one observes shrinking of the condensate. The differences with respect to the standard Thomas-Fermi limit for a condensate without long-range interactions diminish as the number of atoms grows. This is the case because in the $z$ direction the wavefunction is very flat (roughly uniform) and so the integral component almost vanishes. Surprisingly, we observe shrinking of the condensate in the $x$ (repulsive) direction as well. We argue that it is caused by the attraction exerted by very many dipoles concentrated along the soft $(z)$ direction which takes over the repulsive contribution along the $x$-axis. Concluding, in the cigar-shaped trap we observe an overall shrinking of the condensate.

As we see, long-range magnetic interactions do not change qualitatively the ground-state solutions of the Gross-Pitaevskii equation, but they affect mainly a size of the condensate. This indicates that for the used set of parameters an influence of contact (repulsive) interactions is still dominant. However, as we do not know the scattering length for chromium and there are several methods proposed to manipulate this parameter by application of various external fields (e.g. see [10], [11]), we decreased the assumed value and by this means enhanced the effect of long-range (partially attractive) forces. As 
a result, we were able to find a region of unstable solutions. An even more striking discovery was the observation of structured shapes of the wavefunctions acquired by the condensates near the instability threshold. By probing the parameter space $(a, N)$ (scattering length, number of atoms) we found the phase diagram depicting localizations of stable, structured (still stable) and unstable solutions shown in Fig.2. For simplicity we did calculations for a spherical trap with a frequency $\omega_{0}=2 \pi 150 \mathrm{~Hz}$, but a few cases calculated for asymmetric traps convinced us that the qualitative stability behavior would not change. The polarization direction is still $z$. For illustration purposes, we present two examples of the structured solutions. The first one (Fig.3) was obtained for 80,000 atoms with $a / a_{N a}=0.115$ which situates this case very near the instability threshold. The second example (Fig.(1) is a wavefunction for 4,000,000 atoms and $a / a_{N a}=0.233$ (again right above the instability threshold). We have also used geometry of the trap to stabilize solutions unstable in a spherically symmetric potential (by increasing the asymmetry factor $\gamma$ ) as well as to destabilize ones that were stable in a spherical trap (by decreasing $\gamma$ ). It is remarkable that the complexity of the ground-state wavefunction arises solely from atom-atom interactions and does not reflect simplicty of an external potential (it represents self-organization in the ground state).

For all the structured solutions there exists a characteristic length defined by a distance between adjacent maxima in the plane perpendicular to polarization, which is roughly $3 d$. In order to explain this feature, we performed a stability analysis for an infinite (no trap), homogenous case. In this case a proper solution is $\Psi=\frac{1}{\sqrt{V}} \exp (-i g t), V$ being the volume and $g=N \frac{4 \pi \hbar a}{m_{C r}}$. By imposing a small harmonic perturbation of the form $\Psi=\frac{1}{\sqrt{V}}[1+u(t) \cos (\vec{q} \cdot \vec{r})] \exp (-i g t)$ we found that the unstable perturbations are those for which:

$$
q^{2}<\frac{4 N m_{C r}}{\hbar V}\left[\frac{\mu_{0} \mu^{2}}{3 \hbar}\left(1-3 \cos ^{2} \alpha\right)-\frac{4 \pi \hbar a}{m_{C r}}\right]
$$

where $\alpha$ is the angle between the wavevector and the polarization direction. This result implies that surprisingly all the perturbations parallel to polarization $\left(\alpha^{\prime}=0\right)$ are stable, whereas for the direction perpendicular to polarization $\left(\alpha=\frac{\pi}{2}\right)$ there is a long-wave instability. To compare this result with the observed characteristic length of $3 d$ we crudely approximated the volume of our condensates by $V=\frac{4}{3} \pi R_{T F}^{3}$, where $R_{T F}$ is the Thomas-Fermi radius for a condensate without long-range interactions. This yields the critical wavelength:

$$
\lambda_{C}^{2}=\frac{4 \pi^{3} \hbar}{3 N^{\frac{2}{5}} m_{C r}} \frac{(15 a)^{\frac{3}{5}} d^{\frac{12}{5}}}{\left[\frac{\mu_{0} \mu^{2}}{3 \hbar}-\frac{4 \pi \hbar a}{m_{C r}}\right]} .
$$

For the two structured wavefunctions presented in this letter, this value corresponds to $2.2 d$ and $1.8 d$, respectively, which, for such an approximate analysis, is in a surprisingly good agreement. We note in passing that the onset of unstable perturbations $\left(\lambda_{C} \rightarrow \infty\right)$ corresponds to $a_{C} \simeq 0.3 a_{N a}$ which, again, roughly approximates the calculated instability threshold (see Fig.2).

Motivated by experiments under development, all our calculations used chromium parameters. They showed that ground-state solutions differ in a non-trivial way from the usual solutions and found a region of instability as well as structured solutions in its neighborhood. Reaching the interesting region near the instability boundary for chromium seems problematic with stateof-the-art techniques. However, in the case of europium [7] (larger mass and magnetic moment) the instability threshold for 1,000,000 atoms is situated at 92\% of the sodium scattering length value and still grows for larger numbers of atoms (e.g. $108 \%$ for 10,000,000 atoms). Our results may also find use in the blooming area of cold molecules 12 some of which possess large permanent electric dipole moments [13]. For a typical electric dipole moment value of 1 Debye a prefactor of the long-range term is 4 orders of magnitude greater than the corresponding term for magnetic dipole of 1 Bohr magneton. One can thus conclude that behavior of a polar molecular BEC will be overruled by dipole forces.

We would like to thank G.V.Shlyapnikov, M.Brewczyk, Z.Idziaszek and M.Gajda for helpful discussions. K.G. acknowledges support by Polish KBN grant no 203B05715. K.R. and K.G. are supported by the subsidy of the Foundation for Polish Science. T.P. and K.R. acknowledge support by the Alexander von Humboldt foundation. The results have been obtained using computers at the Interdisciplinary Centre for Mathematical and Computational Modeling (ICM) at Warsaw University.

b: current adress: University of Stuttgart, Physics Department, 5th Institute, D-70550 Stuttgart, Germany.

FIG. 1. Half-width of the chromium condensate vs. number of atoms in a cigar-shaped cylindrical trap with $\gamma=0.1$ and $a=a_{N a}$. Filled circles indicate an exact solution for a section along the $x$-axis as compared to the corresponding result in the standard Thomas-Fermi limit (empty circles). Empty squares represent an exact solution along the $z$-axis in comparison with the corresponding standard Thomas-Fermi limit (filled squares). The inset presents analogous results in a disc-shaped trap $(\gamma=10)$ and for $a=a_{N a}$. An exact solution for a section along the $z$-axis (filled squares) is indistinguishable from the standard Thomas-Fermi limit.

FIG. 2. Stability diagram for a spherical trap with $\omega_{0}=2 \pi 150 \mathrm{~Hz}$. Number of atoms is plotted on the horizontal axis and the scattering length (expressed as a fraction of the corresponding value for sodium) on the vertical one. Solid line is the instability threshold - below it no stable solutions could be obtained. The dashed line is the boundary between standard-shaped and structured solutions. In the shaded area structured stable solutions are present. Error bars indicate discreteness of our parameter-space probing. 
FIG. 3. Squared modulus of the ground-state wavefunction in the $x z$ plane for a spherical trap with $\omega_{0}=2 \pi 150 \mathrm{~Hz}, 80,000$ atoms and the scattering length $a / a_{N a}=0.115$. The horizontal axes are in oscillator units $d$.

FIG. 4. Squared modulus of the ground-state wavefunction in the $x z$ plane for a spherical trap with $\omega_{0}=2 \pi 150 \mathrm{~Hz}$, $4,000,000$ atoms and the scattering length $a / a_{N a}=0.233$. The horizontal axes are in oscillator units $d$.

[1] M.H. Anderson et al., Science 269, 198 (1995); K.B. Davis et al., Phys. Rev. Lett. 75, 3969 (1995); C.C. Bradley et al., ibid. 75, 1687 (1995); 79, 1170(E) (1997).

[2] D.G. Fried et al. , Phys. Rev. Lett. 81, 3811 (1998).

[3] Bose-Einstein Condensation in Atomic Gases, Proceedings of the International School of Physics "Enrico Fermi", Course CXL, edited by M. Inguscio, S. Stringari, C.E. Wieman (IOS Press, Amsterdam, 1999), and references therein.

[4] L. Deng et al. , Nature (London) 389, 218 (1999).

[5] L. P. Pitaevskii, 1961, Zh. Eksp. Teor. Fiz. 40, 646 [Sov. Phys. JETP 13, 451 (1961)]; E.P. Gross, Nuovo Cimento 20, 454 (1961).

[6] M. Marinescu and L. You, Phys. Rev. Lett. 81, 4596 (1998).

[7] J. H. Kim et al. , Phys. Rev. Lett. 783665 (1997).

[8] J. D. Weinstein et al. , Phys. Rev. A 57, R3173 (1998).

[9] A.S. Bell et al. , Europhys. Lett. 45, 156 (1999). J. Stuhler et al. (unpublisged).

[10] H. Feshbach, Annals of Physics (N.Y.) 19, 287 (1962); S. Inouye et al., Nature (London) 392, 151 (1998).

[11] P.O. Fedichev, Yu. Kagan, G.V. Shlyapnikov, and J.T.M. Walraven, Phys. Rev. Lett. 77, 2913 (1996).

[12] J.D. Weinstein et al, Nature (London) 395, 148 (1998).

[13] H.L Bethlem, G. Berden, and G. Meijer, Phys. Rev. Lett. 83, 1558 (1999). 
This figure "fig1.gif" is available in "gif" format from: http://arxiv.org/ps/cond-mat/9907308v2 


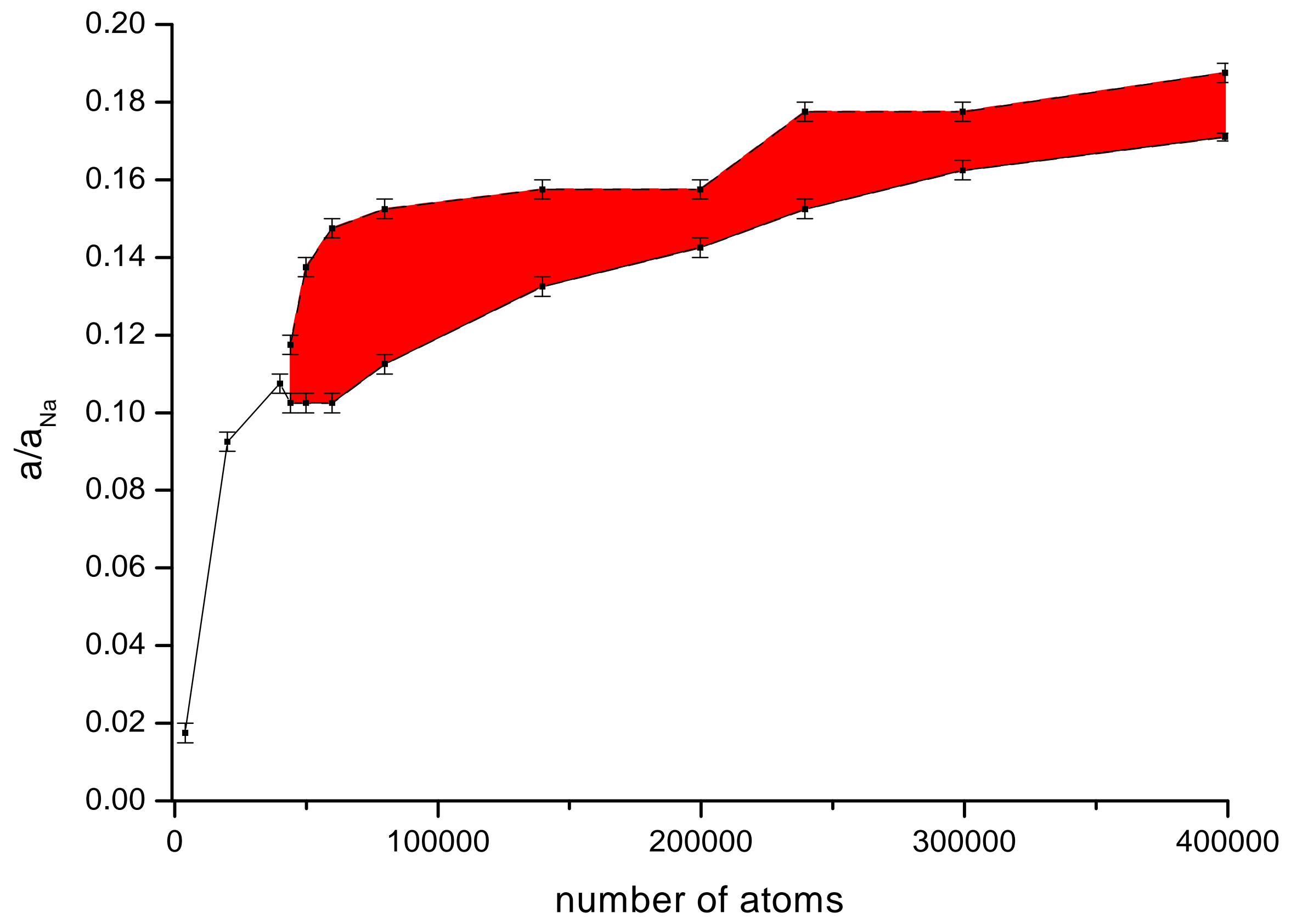


This figure "fig3.gif" is available in "gif" format from: http://arxiv.org/ps/cond-mat/9907308v2 
This figure "fig4.gif" is available in "gif" format from: http://arxiv.org/ps/cond-mat/9907308v2 